\title{
Versuch über die Ökonomie der Schuld. Das radikale Böse in der Darstellung von Holocausttätern: das Beispiel von Peter Schneiders $V_{a t}{ }^{l}$
}

\author{
Essay on the Economy of Guilt. The Radical Evil in the Representation of Holocaust \\ Perpetrators: the Example of Peter Schneider's Vati \\ http://dx.doi.org/10.11606/1982-88372134115
}

\begin{abstract}
This article discusses the question of guilt and penalty in the case of mass murderers as Josef Mengele. The abnormity of the crime, it seems, requires vengeance and not just a punishment restricted to the norms of human rights. In analogy to this assumption, fictional literature on this topic that does not satisfy the reader's desire for revenge, cannot be considered felicitous. The thesis is apparently confirmed by the fate of Vati (1987), a narrative by Peter Schneider which describes the encounter between Mengele and his son in his hideout in Brazil. Concerning the facts based on a series of articles upon the real event, Schneider shows the psychological dilemma of the son who feels unable to deliver his guilty father to the authorities or punish him by his own hands; his failure to punish the perpetrator seems to be compensated by aggressive acts and fantasies against other persons. Schneider has tried to reduce the effect on his audiences, changing the narrator from the first to the third person. Nevertheless, the reception of his book shows that the author did not achieve the proportion between guilt and punishment that would have been expected by the public.
\end{abstract}

Keywords: Nazism; Perpetrators; Josef Mengele; Peter Schneider; Holocaust Literature.

Zusammenfassung: Dieser Artikel diskutiert die Frage von Schuld und Sühne im Fall von Massenmördern wie Josef Mengele. Die Abnormität des Verbrechens, so scheint es, erfordert Rache und nicht eine einfache Bestrafung im Einklang mit den Normen der Menschenrechte. Analog zu diesen Vorüberlegungen kann fiktionale Literatur über das Thema, die das Bedürfnis der Leser nach Rache nicht erfüllt, nicht als gelungen angesehen werden. Diese These scheint bestätigt zu werden durch das Schicksal von Vati (1987), einer Erzählung von Peter Schneider, die das Treffen Josef Mengeles mit seinem Sohn in einem brasilianischen Versteck beschreibt. Hinsichtlich der Fakten, gestützt auf eine Serie von Artikeln über das tatsächliche Treffen, zeigt Schneider das psychologische

\footnotetext{
${ }^{1}$ Der Text wurde ursprünglich vorgetragen am 7. April 2017 in Münchenwiler (Schweiz) auf dem Treffen von swissuniversities, einem Doktorandenprogramm Schweizer Universitäten; das Treffen trug den Titel Un/Schuld.

${ }^{2}$ Universidade de São Paulo, Faculdade de Filosofia, Letras e Ciências Humanas, Departamento de Letras Modernas, Av. Luciano Gualberto, 403, 05508-010, Cidade Universitária, São Paulo, SP, Brasil. Email: hgalle@usp.br
}

Pandaemonium, São Paulo, v. 21, n. 34, maio-ago. 2018, p. 115-133 


$$
\text { Galle, H. - Ökonomie der Schuld }
$$

Dilemma des Sohns, der sich unfähig fühlt, den Vater der Justiz auszuliefern oder ihn eigenhändig zu bestrafen; die fehlende Bestrafung des Täters scheint er durch eine Reihe von aggressiven Akten und Phantasien gegen andere Figuren zu kompensieren. Schneider hat versucht den negativen Effekt auf sein Publikum zu reduzieren, indem er statt des Erzählers in der ersten Person einen Er-Erzähler einführte. Dennoch zeigt die Rezeption des Buchs, dass es dem Autor nicht gelungen ist, das Gleichgewicht zwischen Schuld und Strafe zu finden, das die Öffentlichkeit hätte erwarten dürfen.

Schlüsselwörter: Nazismus; Täter; Josef Mengele; Peter Schneider; Holocaustliteratur.

Der unter der Herrschaft der Nazis systematisch geplante und industriell betriebene Massenmord gilt allgemein als Menschheitsverbrechen, das alles bis dahin Dagewesene und Denkbare überschreitet. Dieser Superlativ bezieht sich weniger auf die absoluten Zahlen der Ermordeten, als auf die brutal-zynische Art der Durchführung eines ideologischen Programms und die absolute Negation des Rechtes auf Existenz für die betroffenen Menschengruppen durch einen Staat unter weitgehender Duldung durch die Bevölkerung. Ein solches Verbrechen sprengt sämtliche Dimensionen, sowohl die des Begreifens und der Darstellung als auch die der Gerechtigkeit. Eine unvorstellbar große Schuld verlangt offenbar auch eine unvorstellbar große Strafe, damit die Waagschalen der Justitia wieder ins Gleichgewicht kommen. Aber wenn Schuld und Strafe unvorstellbar groß sind, gibt es auch kein menschliches Maß, mit dem sie bestimmt werden könnten. Mit den Worten von Vladimir Jankélévitch:

Man kann den Verbrecher nicht mit einer Strafe bestrafen, die zu seinem Verbrechen in einem angemessenen Verhältnis steht: Denn angesichts des Unendlichen neigen alle endlichen Größen dazu, einander auszugleichen, so daß die Bestrafung beinahe gleichgültig wird; das, was geschehen ist, ist im buchstäblichen Sinne unsühnbar. (JANKÉLÉVITCH 2004: 253-254)

Hannah Arendt formuliert diesen übersteigenden Charakter, indem sie schreibt: „Doch das wirklich Böse ist das, was bei uns sprachloses Entsetzen verursacht, wenn wir nichts anderes mehr sagen können als: Dies hätte nie geschehen dürfen.“ (ARENDT 2015: 45). An anderer Stelle spricht sie im Anschluss an Kant vom ,radikal Bösen“, das sich vielleicht daran erkennen ließe, „daß wir es weder bestrafen noch vergeben können, was nichts anderes heißt, als daß es den Bereich menschlicher Angelegenheiten übersteigt und sich den Machtmöglichkeiten des Menschen entzieht“ (ARENDT 2002: 307f.). Jean Améry, der Überlebende der Gestapo-Folter und des Lagers Auschwitz, ging noch einen Schritt weiter: 


$$
\text { Galle, H. - Ökonomie der Schuld }
$$

„Überwältiger und Überwältigte“ könnten erst dann einander begegnen, wenn beide den „Wunsch [...] nach Zeitumkehrung“ hegten: „Die deutsche Revolution wäre nachgeholt, Hitler zurückgenommen“" (AMÉRY 2002: 143). Der Titel von Amérys essayistischem Zeugnis, „Jenseits von Schuld und Sühne“, formuliert in der Paraphrase von Dostojewskis Roman aber auch von Nietzsches Apologie der Immoralität, dass es für die überlebenden Opfer und Täter - wie für deren Nachkommen - ebenso wenig um Rache wie um Sühne gehen kann, weil nicht einmal das jus talionis - also etwa das Aufhängen des Folterknechts Wilhelm Boger in seiner Bogerschaukel - die „ohnehin historisch undenkbare Bereinigung“ (ebd.:142) bewirken könne.

Wenn Améry den Wunsch nach Umkehrung der Geschichte auch auf der Seite der Täter zumindest für denkbar und erstrebenswert hält, so bezieht er sich offensichtlich auf einen moralischen Begriff von Schuld im Sinne der Unterscheidung von Karl Jaspers (JASPERS 1996: 17). Die politische Schuld im engeren Sinne von Regierungs- und Verwaltungsentscheidungen sowie die kriminelle Schuld hinsichtlich der „objektiv nachweisbaren Handlungen, die gegen eindeutige Gesetze verstoßen“ (ebd.), wären allein durch den Wunsch, es hätte nicht geschehen mögen, in gar keiner Weise abzugelten.

So ausgeschlossen die Anwendung des jus talionis tatsächlich ist, wenn es um die Rechtsprechung geht, also um die Bemessung einer Strafe für die einer einzelnen Person zurechenbaren Verbrechen, so unabweisbar erhebt sich die Vorstellung des „Auge um Auge“ vor dem Hintergrund der Exorbitanz der Verbrechen. Zwar gilt die Abschaffung der Todesstrafe den Befürwortern eines humanen Strafrechts als indiskutables Prinzip, aber auch bei seinen dogmatischsten Verfechtern dürfte sich ein Gefühl der Genugtuung darüber einstellen, dass in den Nürnberger Prozessen wenigstens einige der hauptverantwortlichen Politiker und der eifrigsten Folterknechte durch Hängen bestraft wurden. Auf der anderen Seite ist es geradezu unerträglich - das heißt: mit dem Gerechtigkeitsgefühl unvereinbar -, dass so viele andere in die Planung und Ausführung des Genozids Involvierte friedlich in ihren Betten gestorben sind. Philipp Ruch formuliert dies eindrücklich, wenn er zum Verzicht auf Rache an Massenmördern anmerkt:

Die These einer unverlierbaren Würde von Massenmördern wird auch dann durchgehalten, wenn diese die Rechtsfähigkeit Hundertausender außer Kraft gesetzt haben. Ein derartiger moralischer Extremismus ist zumindest diskussionswürdig. Es ist mehr als eine Frage der Logik, ob die Würde eines Völkermörders unverlierbar ist. Selbst heute ist die Vorstellung 


$$
\text { Galle, H. - Ökonomie der Schuld }
$$

schwer zu ertragen, dass die Nürnberger Kriegsverbrecherprozesse mit dem Schutz von Görings Würde geendet hätten und er mit Büchern und Interviews aus dem Gefängnis heraus auch Jahrzehnte später seine Opfer noch hätte verhöhnen und verachten können. (RUCH 2017: 385)

Ganz anders als im wirklichen Leben kommt es in der fiktionalen Darstellung der Nazigräuel in der Regel zu einem Finale, mit dem unser Gerechtigkeitsgefühl befriedigt wird. Zwei besonders erfolgreiche symbolische Repräsentationen des Holocaust aus der jüngeren Zeit scheinen hierfür besonders signifikant, insbesondere da ihr Erfolg offenbar auf einem sehr starken Appell an die Emotionen des Publikums beruht. Ohne Rücksicht auf die rationale, aufgeklärte, politisch korrekte Behandlung, die dem Stoff ,angemessen` wäre, loten sie die Freiheiten der Fiktion aus, um stattdessen einen maximalen kathartischen Effekt hervorzurufen. ${ }^{3}$

Quentin Tarantinos Film Inglorious Basterds (2009) mag bei einigen Zuschauern gemischte Gefühle ausgelöst haben. In meisterhafter Erfüllung der Genreregeln lässt der Regisseur hier durch Helden, die auf der richtigen Seite stehen, sadistische Rachegelüste an extremen Bösewichten befriedigen. Dass es sich bei diesen Bösewichten um die übelsten und gefährlichsten Nazischergen handelt, legitimiert ihre Eliminierung nicht nur fiktionsintern, sondern auch historisch. Christoph Waltz spielt nicht nur die im Drehbuch vorgesehene Rolle des zynischen Schurken, er verkörpert zugleich jenen Typus des gebildeten und eiskalten Karrierenazis, der in den vergangenen Jahren durch Studien wie Michael Wildts Generation des Unbedingten (2008) herausgearbeitet wurde. Eine solche Figur verdient keine „mildernden Umstände“, nicht vor Gericht und schon gar nicht im Film. Anders als im Mainstream, kommen die Nazitäter allerdings nicht in einer finalen Explosion oder dergleichen um, sondern sie werden genussvoll und persönlich einer besonders barbarischen und schmerzhaften Behandlung unterzogen, indem ihnen die (jüdisch-amerikanischen)

\footnotetext{
${ }^{3}$ Der Begriff der Katharsis wird hier nicht im strengen Sinne von Aristoteles ‘ Poetik verstanden, wo er an sehr spezifische Handlungstypen geknüpft ist. Für Aristoteles wird bei den Zuschauern die Reinigung (Katharsis) von den Emotionen Schrecken und Mitleid durch die Darstellung eines sittlich nicht verbrecherischen Menschen hervorgerufen, der durch eine Verfehlung ins Unglück stürzt. Dass ein Verbrecher mit Glück belohnt wird (tendenziell der Fall Mengeles), wäre für Aristoteles eine dem Tragischen entgegengesetzte Handlung. Er betrachtet jedoch eine Fabel wie die des Odysseus, wo die Guten belohnt und die Bösen bestraft werden, immerhin als die „Zweitbeste“ Möglichkeit für eine Tragödie. „Sie gilt [manchen] als die beste, weil sie der Schwäche des Publikums entgegenkommt“ (ARISTOTELES 1994: 41). Vgl. zu Aristoteles SEIDENSTICKER (2009) und zu einer alternativen Konzeption von Katharsis HuG (2004).
} 


$$
\text { Galle, H. - Ökonomie der Schuld }
$$

Vollstrecker der ausgleichenden Gerechtigkeit bei lebendigem Leibe den Skalp abziehen. (Tarantino weiß übrigens um die große Beliebtheit dieser indianischen Kulturtechnik gerade bei den deutschen Lesern von Karl Mays Wild-West-Romanen, zu denen bekanntlich Adolf Hitler gehörte. $)^{4}$ Es scheint, dass Tarantino in diesem wie in anderen Filmen (Django Unchained, 2012) auf eine höchst intelligente Weise mit anthropologisch unvermeidbaren Reaktionen spielt und sie gewissermaßen in den richtigen historischen Kontext einbringt. Das kann natürlich ein erhebliches Befremden über die eigenen Gefühle bei Zuschauern auslösen, die gerade diese emotionale Mechanik von Produkten der Kulturindustrie für gefährlich halten. Die archaischen emotionalen Reaktionen scheinen hier umso gefährlicher, als sie durch das geschichtliche Element im Plot legitimiert werden.

Scheinbar in die entgegengesetzte Richtung gehen Bernhard Schlinks Roman Der Vorleser von 1995 und der von Stephen Daldry nach dieser Vorlage gedrehte Film von 2008. Der immense Erfolg (der größte internationale Bestseller seit Grass‘ Blechtrommel und Süskinds Parfüm) beruht wie bei Tarantino vor allem auf der emotionalen Wirkung der durchkonstruierten Fiktion und nicht auf irgendeiner Anlehnung an historische Figuren und Ereignisse. Die ausgelöste emotionale Identifikation richtet sich aber nicht auf die ihren Rachegelüsten nachgehenden Vertreter der Opfer, sondern auf eine Täterin. Dem Autor Schlink gelingt das Kunststück, den Leser Empathie mit einem Mitglied des Wachpersonals von Auschwitz empfinden zu lassen, allerdings nur dadurch, dass einerseits die persönlich zurechenbare Schuld aufgrund der individuellen Bedingungen so gering wie möglich gehalten wird (Unterschicht, Frau, Analphabetin, Befehlsempfänger auf unterster Ebene der Hierarchie) und dass andererseits diese unfreiwillig zur Täterin Gewordene eine höhere Strafe auf sich nimmt, als ihr aufgrund des positiven Rechts zukäme. Sie tut dies zwar anfangs aus Scham, denn sie will verbergen, dass sie den fraglichen Bericht über das ,Massaker‘ (eigentlich zur Hälfte ein Unglücksfall) aufgrund ihres Analphabetismus gar nicht hätte verfassen können, aber die Scham verwandelt der Autor in ein echtes Bewusstsein von Schuld und Reue, indem er sie in der Haft freiwillig die kanonischen Berichte von Überlebenden lesen und vor der Entlassung schließlich Selbstmord begehen lässt. Wenn eine Mehrzahl von Lesern diese Ökonomie von (relativ) geringem Anteil an persönlicher Schuld

\footnotetext{
4 Vgl. Scholz, Martin. „Bumm, bumm, bumm, bumm“. Quentin Tarantino im Interview. Frankfurter Rundschau, (03.08.2009). 


$$
\text { Galle, H. - Ökonomie der Schuld }
$$

und (relativ) großer Buße für überzeugend hält, spricht das auch in diesem Fall dafür, dass in Bezug auf die Naziverbrechen ein eigenartiger irrationaler Proporz waltet: Zwar lässt sich das Überdimensionale des Gesamtverbrechens eigentlich durch keine wie immer geartete Sühne ,wiedergutmachen“ und verlangt nach einem Exzess von Bestrafung, doch lässt sich auch bei einer direkten Beteiligung am Morden durch eine gezielte und kalkulierte Minimierung des individuellen Anteils und eine moralische Einsicht in die Schuld tatsächlich eine Art von Gerechtigkeit herstellen oder besser gesagt: Unser Gerechtigkeitsgefühl wird durch diese Fiktionen befriedigt.

Wie gesagt, nur in der Fiktion. Es ist zu Recht beanstandet worden, dass Schlinks Protagonistin keinem aktenkundig gewordenen Verlauf von Täterbiographien entspricht und eine freie, wenn auch sehr effiziente Konstruktion ist (ADLER 20.04.2002; COLLINS Donahue 2001; Collins Donahue 2010). Wie bei Tarantino handelt es sich also gewissermaßen um eine kontrafaktische Wunschvorstellung, die zu einer in sich stimmigen literarischen bzw. filmischen Fiktion ausformuliert wurde. Aus dieser Art von Werken lernt man garantiert nichts über die Vergangenheit, wie sie wirklich war, aber ihr kathartischer Effekt ist gewaltig und das Übermaß an ungesühnter Schuld wird noch viel mehr Produkte dieser Art zeitigen.

Unter den am schwersten belasteten Figuren des Holocaust nimmt Josef Mengele eine zentrale Position ein. Er hat nach dem Zeugnis vieler Überlebender nicht nur an der Rampe und in den Krankenbauten die Opfer der Gaskammern selektiert, sondern mit eigener Hand getötet, Kinder und Frauen durch brutalste Manipulationen zusätzlich gequält und dadurch absichtlich unsägliches Leid verursacht. Erschwerend kommt hinzu, dass Mengele als Mediziner an den hippokratischen Eid gebunden war und in der Rolle des Arztes auf viele seiner Opfer zunächst Vertrauen erweckend gewirkt hat. Als überzeugter Nazi hat er sich nach diesen Verbrechen einer Strafverfolgung entzogen und sowohl unter seiner echten als auch unter gefälschten Identitäten in Südamerika bis zu seinem Tod in Freiheit leben können, ohne seine Anschauungen zu irgendeinem Zeitpunkt zu revidieren oder Reue zu empfinden. Emblematischer Höhepunkt von vielen Zeugenberichten, ist er zugleich die Inkarnation des dämonischen Nazitäters, der noch in unserer Nachkriegsgegenwart seine teuflischen Ziele verfolgt (The Boys from Brazil, Wakolda). Wie keinen zweiten verbindet ihn das kollektive Imaginäre der Menschheit mit der Schuld am Massenmord. 


\section{Galle, H. - Ökonomie der Schuld}

Gleichzeitig wissen wir, dass er Zeit seines Lebens nicht bestraft wurde, sondern als freier Mann in Brasilien leben konnte, was bei den meisten Mitteleuropäern sehr wahrscheinlich - trotz des ambivalenten Bildes von Lateinamerika in den Medien - eher positive Vorstellungen weckt, die mit Tropen und angenehmem Leben verknüpft sind, also dem Gegenteil einer Strafe. Wenn sich ein Autor dieser Figur annimmt, sollte also die im Vorangegangenen skizzierte „Ökonomie“ von Schuld und Bestrafungswunsch eine Rolle spielen. Es soll versucht werden, an Peter Schneiders Erzählung Vati genau dies zu zeigen.

\section{Peter Schneiders Erzählung Vati}

Eine Weile bevor sich Peter Schneider in der Studentenrevolte radikalisierte und dann mit seiner ersten Novelle Lenz berühmt wurde, veröffentlichte er in der damals als antikommunistisch verschrienen Berliner Zeitschrift Der Monat einen Artikel über „Politische Literatur“ (SCHNEIDER 1965), in dem er sich fragte, ob eine „dichterische Beschreibung des Dritten Reiches, die Anspruch aufs politische Detail macht“, prinzipiell missglücken muss, sei es weil sie als Fiktion die politische Realität verfehlt, oder weil sie als Dokumentation die Literarizität einbüßt. An Brechts Arturo Ui führte er vor, wie selbst ein begabter Autor die Komplexität des Historischen verfälschen muss, wenn er es in fiktionaler Form bühnenfähig konzentrieren will. Als Beispiel für Dokumentarismus nennt er Heinar Kipphardts Stück In der Sache Robert J. Oppenheimer (1964), das im Vorjahr aufgeführt worden war. Auch wenn man seinem negativen Urteil über Kipphardt folgen will, kann es wohl kaum für Peter Weiss` Ermittlung gelten, die zwei Monate vor Erscheinen des Artikels uraufgeführt wurde und neue Maßstäbe für Dokumentarismus und Poetizität setzte, von Schneider aber sehr wahrscheinlich nicht mehr in seine Reflexionen einbezogen werden konnte. In jedem Fall hat er in dem Artikel sein Gespür dafür ausgedrückt, dass der Komplex des Nazismus sich tendenziell der fiktionalen Darstellung entzieht.

Diese Position hat er später wenigstens durch die schriftstellerische Praxis revidiert. Gut zwanzig Jahre später nämlich veröffentlichte er die Erzählung Vati (1987), in der er sich am Themenkomplex des Massenmords auf halb fiktionale, halb dokumentarische Weise versuchte und - nach recht einhelliger Ansicht der damaligen Rezensenten - scheiterte. Einer 


\section{Galle, H. - Ökonomie der Schuld}

der beiden Haupteinwände war die - anfänglich nicht gekennzeichnete - Übernahme von Textpartien aus gedruckten Quellen, ${ }^{5}$ vor allem aus einer umfangreichen Artikelserie der Bunten Illustrierten von Inge BYHAN (1986) ${ }^{6}$ über Josef Mengele und den Besuch seines Sohnes Rolf in Brasilien - also gerade der dokumentarische Zug des Textes. Heftigere Kritik als die Plagiatsfrage erregte jedoch das Thema an sich und seine literarische Ausführung. So äußerte Gerda-Marie ScHÖNFELD (1987) im Spiegel, „daß sich der Autor Peter Schneider anhängt an einen Nazi-Papi, ist obszön und ärgerlich genug“‘, und behauptete weiter, ,beim Thema Mengele“ sei es unvermeidlich zu mystifizieren: „Sich aus fiktiver SohnesPerspektive anzunähern an ein Phantom des Grauens, kann nur anbiederisch werden.“ Andere Rezensenten fühlten sich an Kohls Auftritt in Bitburg erinnert und schämten sich, oder sie warfen dem Autor Kitsch vor (vgl. KRASS 2012: 99). Vorangegangen war diesem Eklat schon eine ähnliche Erfahrung Schneiders auf einer Lesereise durch die USA, wo er zunächst in Berkeley vor Germanisten die später publizierte Fassung mit Ich-Erzähler vortrug und erhebliches Missfallen auslöste; man warf ihm vor, dass der Text die „Fakten von Mengeles Verbrechen ausspare und letztlich ihrer Verharmlosung diene“ (SCHNEIDER 2011: 149). Er veränderte daraufhin die Erzählperspektive und ließ die Geschichte in der dritten Person weitgehend im Konjunktiv Präsens mitteilen, mit der Folge, dass sie bei weiteren Lesungen in den USA nicht mehr auf Widerstand stieß. Der Autor selbst (SCHNEIDER 2011: 151f.) schreibt diesen Effekt einer stärkeren Distanzierung durch den heterodiegetischen Erzähler zu und bedauert, damals seinen Verleger nicht davon überzeugt zu haben, die revidierte Fassung zu drucken, und so die negative Rezeption seines Buches zu vermeiden. Ob dies tatsächlich so geschehen wäre, ist allerdings fraglich und ließe sich möglicherweise durch eine empirische Studie klären. Denn gemäß der hier vertretenen These wäre es weniger die Erzählstruktur als vielmehr der Plot und das Ausbleiben der Strafe, die beim Leser ein unbehagliches Gefühl zurücklassen.

\footnotetext{
${ }^{5}$ In der später publizierten Taschenbuchausgabe (1996: 64) werden am Ende des Buches die Artikelserie von Inge Byhan und drei weitere Quellen genannt, aus denen einige Redeweisen der handelnden Personen und Handlungsdetails in die Erzählung Vati übernommen sind.

${ }^{6}$ Die Bunte Illustrierte gehörte auch damals nicht $\mathrm{zu}$ den unbedingt $\mathrm{zu}$ den als „Qualitätsmedien“ zu bezeichnenden Zeitschriften, sondern eher zur „Regenbogenpresse“. Dass sich Rolf Mengele ausgerechnet an dieses Blatt gewandt hatte, um seinen Besuch in Brasilien sieben Jahre nach dem Tod seines Vaters öffentlich zu machen, warf ein schiefes Licht nicht nur auf ihn selbst, sondern auch auf Peter Schneider, der sich dieses reißerisch aufgemachten, mit vielen großen Fotos illustrierten Materials bediente.
} 


\section{Galle, H. - Ökonomie der Schuld}

Zunächst ist zu konstatieren, dass der Autor sich sehr eng an die dokumentierten Fakten gehalten hat. Er selbst betont, es wäre ihm bei den Übernahmen aus der Zeitschrift vor allem um die „Redeweisen“ der Figuren gegangen. Das ist erkennbar, aber er wollte offenbar auch in die Grundkonfiguration des Plots wenig eingreifen: Der Name Mengele wird nicht genannt und die der übrigen Beteiligten werden verändert, doch die biographischen Konstellationen von Familie und Freundeskreis in Brasilien entsprechen fast eins zu eins der inzwischen bekannten Situation: Da ist Mengeles Sohn Rolf aus der kurz nach dem Krieg geschiedenen Ehe, und dessen Aufwachsen in dem Glauben, er habe einen Onkel in Südamerika, um dessen Gunst er mit dem Cousin Karl-Heinz (im Buch) konkurriert, dem Sohn von Martha Mengele, Witwe eines Bruders von Josef, der sie in den fünfziger Jahren in Buenos Aires heiratete. Nach Jahren eines sporadischen und distanzierten Briefwechsels zwischen Vater und Sohn dann die halbwegs konspirative Reise nach Brasilien, einige Tage in dem einfachen Haus Mengeles in der Vorstadt und ein paar Treffen mit dem befreundeten Ehepaar Bossert (im Buch: Weinert), bei dem es nicht zu einer echten Annäherung, aber auch nicht zum definitiven Eklat zwischen Josef und Rolf kommt und schließlich zwei Jahre später der Tod Mengeles beim Baden im Meer.

Angesichts der nur ein Jahr zurückliegenden Artikelserie in der Bunten Illustrierten, war den Lesern klar, dass es sich nicht um beliebige, freie erfundene Figuren handelt, sondern - fast wie in einem Schlüsselroman - um eine Fiktionalisierung des Besuchs von Rolf Mengele bei seinem Vater in São Paulo, um den Versuch also, den seelischen Konflikt eines Generationsgenossen von Peter Schneider, der mit einem derartig schuldbeladenen Vater konfrontiert ist. Der junge Mann steht im Fokus der Erzählung, der Vater bleibt in seiner Wahrnehmung schemenhaft und fremd.

Dass der fiktive (wie der reale) Mengele jede persönliche Schuld rundweg abstreitet und sich auf den Befehlsnotstand beruft wie fast alle seine Genossen, dabei jedoch zugleich die wahnhafte Rassenideologie propagiert, die gerade die abgestrittenen Taten rechtfertigt, gehört zu den geläufigen Paradoxien revisionistischer Rede von Alt- und Neonazis. Man darf davon ausgehen, dass seine Verteidigungsstrategie vor Gericht nicht viel anders gewesen wäre. Er äußert kein Wort des Bedauerns oder der Reue über die geschehenen Verbrechen. Schon vor Jahren hatte er dem Sohn in einem Weihnachtsbrief geschrieben - und Schneider übernimmt das fast wörtlich: „Ich nehme zur Kenntnis, daß ich Respekt und Verständnis für 


$$
\text { Galle, H. - Ökonomie der Schuld }
$$

meinen Lebensweg von dir nicht erhoffen kann. Andererseits sehe ich nicht die geringste Veranlassung, Dir für irgendwelche Entscheidungen, Handlungen oder gar für meine Philosophie Rechenschaft abzulegen!“ (SCHNEIDER 1996: 20). In der Erzählung und in den Berichten Rolf Mengeles bekräftigt er die Beteuerung seiner persönlichen Unschuld mit einem einigermaßen grotesken Schwur, der sich im Wortlaut der Biographen POSNER und WARE (1993: 338) so ausnimmt: „Beim Augenlicht meiner Mutter, ich habe nie jemandem etwas zuleide getan.“ Auch gegenüber der Journalistin Inge Byhan hatte der Sohn die Worte zitiert, sein Vater habe ihm ,,in einer langen Nacht in São Paulo ,beim Augenlicht meiner Mutter ${ }^{6}$ geschworen [..], daß er ,nie jemanden getötet, nie jemandem persönlich etwas zuleide getan hat““ (BYHAN 1986b: 26). Peter Schneider (1996: 29) zitiert diesen Schwur, allerdings unter Veränderung des Possessivpronomens: hier schwört Mengele „beim Augenlicht deiner Mutter", wodurch die garantierende Instanz von der Erzeugerin Josef Mengeles auf die Mutter Rolfs verlagert wird. Das ist aus der Perspektive des Vaters psychologisch wenig überzeugend (warum sollte er sich auf seine erste Frau berufen, von der er seit Jahrzehnten geschieden ist), passt aber als persuasive Strategie in die kommunikative Situation.

Was die objektive, justiziable Schuld Josef Mengeles angeht, hätte diese in einer gültigen Form von einem Gerichtsverfahren mit unabhängigen Richtern festgestellt werden müssen, was Mengele lebenslang vermieden hat. Von seinem Sohn darauf angesprochen, behauptete er, „daß es für ihn keine Richter gäbe, nur Rächer“(VÖLKLEIN 1999: 299; BYHAN 1986a: 32II; in wörtlicher Rede bei SCHNEIDER 1996: 44). In der „Weltanschauung“, der Mengele bis zum Schluss anhing, besteht die Natur- und Weltgeschichte aus einem ewigen Kampf, in dem sich der Stärkere zwangsläufig durchsetzen muss; die Niederlage des Nazismus ist für ihn nur eine vorläufige und wird vom Lauf der Geschichte korrigiert werden. Wenn sein Sohn glaubt, der Vater würde in der Gegenwart gerechte Richter finden, so geht er von einem grundsätzlich anderen Verständnis von Gerechtigkeit aus, das auf dem Prinzip der Gleichheit aller Menschen und der Gleichberechtigung der Völker basiert. Für Josef Mengele sind das falsche Prämissen, und so ist es konsequent, dass er sich versteckt hält, und ebenso, dass er seine Handlungen nicht bereut. Er schwört, um seinen Sohn von seiner „Unschuld“ zu überzeugen, wie er offenbar seine übrige Familie und den Prokuristen Sedelmeier überzeugen konnte, die ihn über all die Jahre unterstützt haben. 


$$
\text { Galle, H. - Ökonomie der Schuld }
$$

Interessanterweise ist das Bild des historischen Mengele widersprüchlich. Unter den zahllosen Zeugenaussagen über Mengeles Verhalten als KZ-Arzt gibt es einzelne, die Mengele als pflichtbewussten, korrekten und sogar fürsorglichen Menschen schildern. Diese Beispiele sollen hier ausführlicher zitiert werden, gerade weil das öffentliche Bild von Mengele so ausschließlich von der ,Bestie ' geprägt ist. Es ist in der Tat irritierend, aber ein Faktum, dass der skrupellose Massenmörder selbst im Kontext des Lagers einen positiven Eindruck hinterlassen konnte.

Hermann Langbein, selbst Häftling in Auschwitz und später intensiv mit der historischen Erforschung befasst vermerkt in seinem Buch Menschen in Auschwitz:

Menschen, die Mengele näher kannten, beschreiben ihn nicht als einen sadistisch veranlagten Wüterich. Czelny hebt hervor, daß er mit Häftlingen immer in höflicher Form und betont ruhig sprach und die bei der SS üblichen Grobheiten unterließ. [...] Eine polnische Pathologin, die für ihn Untersuchungen durchzuführen hatte, wurde mit seiner Hilfe aus Auschwitz entlassen, da sie schwanger war. Als Freigelassene mußte sie in Krakau weiter für Mengele Gewebeschnitte anfertigen. Nach der Entbindung schickte ihr Mengele einen Blumenstrauß. (LANGBEIN 1999: 502)

Das Zeugnis von Miklós NyISZLI (2005), der 12 Monate lang die von Mengele geschickten Leichen obduzieren musste, beschreibt ihn als ruhige, überlegt handelnde Person, die aus Überzeugung Tausende in die Gaskammer selektierte und für seine eigenen Experimente Zwillinge und Häftlinge mit physischen Besonderheiten töten ließ; andererseits erlaubte ihm Mengele, sich um Frau und Tochter zu kümmern, so dass er sie auf einen „rettenden“ Transport in ein Arbeitslager bringen konnte. Die slowakische Häftlingsärztin Margita Schwalbova hatte laut VÖLKLEIN (1999: 22) „keine Kenntnis davon, daß Dr. Mengele selbst Leute umgebracht hat“. Derselbe Autor bemerkt auch: „Daß Mengele medizinische Eingriffe nicht persönlich durchgeführt habe, ja sich überhaupt den Gefangenen und Kranken möglichst fernhielt, bestätigen einige der überlebenden Häftlingsärzte und Pfleger“ (1999: 19). Aussagen dieser Art mögen den Eindruck verstärkt haben, dass es sich bei Mengele zwar um einen linientreuen nazistischen Massenmörder und besessenen Forscher gehandelt habe, aber auch um einen sich in bestimmten Situationen „,anständig“ verhaltenden Menschen. Es ist daran zu erinnern, dass Himmler diesen Ausdruck verwendete, um in seiner Posener Geheimrede vor SS-Führern zu betonen, dass die Durchführung des Massenmordes nicht zu einer allgemeinen Verrohung der Ausführenden 


$$
\text { Galle, H. - Ökonomie der Schuld }
$$

geführt habe: man konnte Tausende Frauen, Kinder und Greise erschießen, vergasen und verbrennen lassen, ohne darauf zu verzichten, sich selbst als ,anständige“ Menschen zu empfinden, wenn man nur nach dem Massaker seine guten Manieren behielt. Mengele mag sich selbst ebenso gesehen haben, was die autobiographischen Aufzeichnungen nahelegen, die er in Brasilien anfertigte und in denen er geradezu als Opfer erscheint (vgl. GALLE 2011).

Es gibt freilich auch sehr viele Zeugenberichte, die Mengele in einem völlig anderem Licht zeigen, nämlich als ,Exzesstäter ${ }^{6}{ }^{7}$ der in cholerischen Anfällen Menschen eigenhändig erschoss, die ihm im Weg standen, schwangere Frauen und Neugeborene schwer misshandelte und Giftinjektionen in Augen und Herzen vornahm. Man kann diese Aussagen weder beweiskräftig verifizieren, noch kann man all diese Berichte, die ihm den Beinahmen „Todesengel“" verschafften, als fehlgehende Erinnerungen oder Verwechslungen mit anderen Ärzten vom Tisch wischen. Josef Mengele dürfte im Lager diese beiden Gesichter gehabt haben und sein Persönlichkeitsprofil weist noch dreißig Jahre später zwiespältige Züge auf: charmant und einnehmend einerseits, rechthaberisch und streitsüchtig andererseits. Und selbst wenn er nicht ein einziges Mal selbst Hand angelegt hätte bei all den von ihm durchgeführten Experimenten: Man darf vermuten, dass jedes alliierte Gericht nach 1945 ihn aufgrund der Menge an Zeugenaussagen und Beweisen (dazu zählen auch die nach Dahlem und Straßburg geschickten Präparate von getöteten Menschen) für schuldig befunden und die Höchststrafe verhängt hätte. Schon seine Funktion als Lagerarzt, die Selektionen und die Anordnung von Tötungen würden nach den Rechtsvorstellungen heutiger Demokratien völlig ausreichen, um ihn $\mathrm{zu}$ verurteilen, ganz $\mathrm{zu}$ schweigen von den medizinischen Experimenten. Eichmann wurde in Jerusalem für seine Dienste in der Verwaltung gehenkt, und für ihn konnte - zumindest in einem formalen, technischen Sinn - eher als für Mengele gelten, dass er „nie jemandem persönlich etwas zuleide getan“ habe (BYHAN 1986b: 26). Und wenn Mengele seinem Sohn gegenüber „beim Augenlicht“ (ebd.) seiner Mutter schwört: Welche Bedeutung hat die Übertretung des achten Gebots (falsch Zeugnis ablegen) für einen Mann, der sich so souverän über das sechste (du sollst nicht töten) hinwegsetzen konnte? Das fragte sich schon Hannah Arendt.

\footnotetext{
${ }^{7}$ Exzesstaten sind Wolfgang Sofsky zufolge ohnehin keine Ausnahmen, die nur von psychisch besonders disponierten SS-Leuten ausgeführt werden, sondern systematisch im Rahmen der totalitären Gewalt des Lagers auftretenden Ereignisse, an denen prinzipiell alle Inhaber der Macht beteiligt sein können (vgl. SOFSKY 1997: 255).
} 


$$
\text { Galle, H. - Ökonomie der Schuld }
$$

In der Erzählung erscheinen die Taten Mengeles nur als Phantasie des Sohnes. Am Abend nach dem ersten aufreibenden Gespräch mit seinem Vater will er aus der beklemmenden Atmosphäre der Hütte entkommen, aber bevor er zur Tür kommt, steht dieser plötzlich hinter ihm und wirkt nun nicht mehr wie ein betulich-sentimentaler Rentner, sondern wie ein gefährliches Raubtier.

Unwirklich groß und mächtig stand er vor mir. Ich sah seine kräftigen, behaarten Arme in dem kurzärmligen Hemd, ich erkannte eine entsetzliche Kraft in diesem Körper, etwas Unberechenbares, Gewalttätiges, und plötzlich schob sich mir - scharf und deutlich, als könnte ich selbst diese Vorgänge bezeugen - ein Bild vor die Augen. Ich sah einen gepflegten, immer ruhigen Mann in SS-Uniform, der, durch eine einzige unerlaubte Bewegung zu wahnsinniger Wut gereizt, ohne Vorwarnung den Kopf eines Gefangenen mit dem Knauf seiner Dienstpistole zerschlägt oder mit seinen Stiefeln den Bauch einer Schwangeren zertritt. (SCHNEIDER 1996: 34-35)

Für diese Episode gibt es kein Vorbild in den Artikeln zu Rolf Mengeles Besuch, sie ist Bestandteil der Fiktionalisierung. Wohl aber handelt es sich um Taten, die dem KZ-Arzt in Zeugenberichten zugeschrieben wurden. Sie werden hier nicht entkräftet dadurch, dass es sich um eine Art von Sinnestäuschung handelt und nicht um einen wirklichen Angriff - in der Folge wird der Vater nun nichts Gewalttätigeres tun, als mit zitternden Händen seine „viel zu schweren Schuhe abzustauben“ (SCHNEIDER 1996: 35). Die Erscheinung des Vaters ist in sich zweideutig, sie ermöglicht, ihn in exakt der Rolle vorzustellen, die ihm das kollektive Imaginäre im Laufe der Jahrzehnte zugeschrieben hat. Ohne selbst über den Wahrheitsgehalt dieser Zuschreibungen zu entscheiden, legt der Autor nahe, sie für möglich zu halten, auch wenn die aktuelle Figur mit ihren 65 Jahren einen anderen Anschein erweckt und erwecken will. Der Autor hätte auch die konkreten Stimmen von Überlebenden zitieren können, um Mengeles Vorleben in die Fiktion einzubringen, aber das hätte die Unsicherheit des Sohnes, der allein mit seinem Vater konfrontiert ist, nicht getroffen. In einer früheren Manuskriptversion findet sich der Hinweis auf die Mengele konkret zugeschriebenen Greueltaten an einer anderen Stelle, dem Kreuzverhör, in dem er seinem „Vater Fragen [stellt], die niemals zuvor ein Sohn seinem Vater zu stellen gezwungen war“" (SCHNEIDER 1996: 28). „Hatte er, ja oder nein, zwei Zwillingspaare mit seiner Dienstpistole erschossen, einen Neugeborenen lebend ins Feuer geworfen, einen Säugling unter den Augen der Mutter bei lebendigem Leibe seziert, dieses und Anderes, Unaussprechliches hörte ich mich fragen“ (Manuskript C, Mappe 1519, S. 16). 


$$
\text { Galle, H. - Ökonomie der Schuld }
$$

Die Lösung in der endgültigen Version ist m. E. literarisch überzeugender, weil sie den unsicheren epistemischen Status dieser vergangenen Ereignisse als Imagination inszeniert. Zugleich ist sie jedoch emotional unbefriedigender, denn in unserer Vorstellung verlangt das ungeheure Verbrechen nach dem ungeheuren Verbrecher und einer ungeheuren Strafe, während in der Erzählung nichts dergleichen auftaucht. Der fiktionale Mengele ist eben nicht mehr als ein rassistische Phrasen schwadronierender Querkopf, der vergeblich versucht, seinen Sohn von seinen Irrlehren zu überzeugen und insgesamt eine ziemlich erbärmliche Figur macht, womit Schneider sich stark an der Darstellung von Rolf Mengele in der Bunten Illustrierten orientiert.

Der fiktionale Sohn als Protagonist und Erzähler schafft es nicht, einen wirklich festen Standpunkt zu gewinnen. Zwar hält er die Vorträge seines Vaters für gefährlichen Unsinn, der schon an sich nach einer Strafe verlangt:

Verbieten, ausrotten! Man muß ihm das Denken und Sprechen verbieten. Hilflos der Strafund Schuldbegriff, der nach dem individuellen Tatanteil, nach niedrigen Beweggründen fragt: diese Art Mörder sind die harmlosen, die zu umarmenden Mörder. Man muß die mörderischen Gedanken bestrafen. (SCHNEIDER 1996: 56)

Das entspricht dem Bedürfnis des Lesers, doch es bleibt wieder folgenlos. Befangen in dem Dilemma, dass er, wollte er den Massenmörder bestrafen und die Opfer rächen, seinen eigenen Vater töten oder zumindest verraten müsste, tritt der Sohn erneut die Flucht an. Der Vatermord wäre zwar in der griechischen Antike weniger schuldbeladen als die Tötung der Mutter, aber ein Atriden-Schicksal wäre es allemal, auch heute noch. Es wäre nicht realistisch, aber kathartisch.

Seine Flucht vor dem Vater führt ihn, wie schon am ersten Abend, in die brasilianische Realität, und zwar in die Favela-artig geschilderten Bezirke des tropischen Belém, während die reale Begegnung in einem armen südlichen Vorort der Megalopolis von São Paulo stattgefunden hat. $^{8}$ Diese beiden Ausflüge aus dem deutschen

\footnotetext{
${ }^{8}$ Im Interview mit Inge Byhan beschreibt Rolf Mengele selbst den Weg in das Viertel, in dem das Haus, „eigentlich eher eine Holzhütte“, seines Vaters stand, als Weg durch „Elendsquartiere, kilometerlang, die berüchtigten Favelas“. (BYHAN 1986a: 6). Zumindest aus heutiger Sicht ist das Viertel Eldorado im Munizipium Diadema (über 400.000 Einwohner, zwischen São Paulo und São Bernardo dos Campos gelegen) sicherlich keine Favela, wenn auch ein eher armer und abgelegener Bezirk. Durch die dortigen Stauseen entsteht sogar ein gewisser Freizeitwert. Das Haus Mengeles soll nach dessen Tod 25.000 Dollar wert gewesen sein, für Brasilien Ende der 1970er Jahre keine ganz unbeträchtliche Summe (POSNER / WARE 1993: 352).
}

Pandaemonium, São Paulo, v. 21, n. 34, maio-ago. 2018, p. 115-133 


$$
\text { Galle, H. - Ökonomie der Schuld }
$$

Schuldzusammenhang in das brasilianische Großstadtleben sind die wichtigsten fiktionalen Erweiterungen, die Peter Schneider dem dokumentierten Verlauf der Reise und der Auseinandersetzung Rolf Mengeles mit seinem Vater hinzugefügt hat. Der Autor muss also Gründe gehabt haben, sie zu erfinden. In beiden Episoden bewegt sich der Sohn selbst als weißer Europäer wie der Vater in einer Umgebung von ,halbnackten, dunkelhäutigen Nachbarn“", in einer Gesellschaft, die von Korruption und staatlicher Willkür geprägt ist. Und in beiden Episoden wird der Sohn auf eine Art und Weise ,schuldig“, die nicht durch die konkrete Handlung des Abschnittes motiviert ist. Die Aggressionen, die sich eigentlich gegen den Vater richten müssten, entwickelt er gegenüber Personen, die beschrieben werden als Mitglieder einer - verglichen mit dem weißen europäischen Touristen - sozial schwachen Schicht in einem Randbezirk von Belém. Tendenziell und unwillkürlich reproduziert Rolf Mengele dabei das Rollenmuster, das die Nazis seinem Vater ermöglicht hatten und das diesen Vater schuldig werden ließ.

Der erste Gang durch die nächtliche Großstadt lässt den Sohn einem hochgewachsenen Mädchen folgen, das zuerst durch eine Reiseschreibmaschine sein Interesse und bald darauf durch ihre Attraktivität seine „Gier“ weckt. Durch ,,ihren dunklen Nacken und ihre nassen, bis zum Oberschenkel nackten Beine“ (SCHNEIDER 1996: 36) ist sie als Einheimische gekennzeichnet, die ihn, wie er meint, als „Angehörigen einer anderen Rasse“ sehen dürfte (SCHNEIDER 1996: 38). Wie unter Zwang folgt er der fremden jungen Frau, ohne jedoch seinen widersprüchlichen Impulsen nachzugeben (,Sie in einen Hausflur zerren, ihr die Kleider vom Leib reißen oder mich vor ihr auf die Knie werfen“; SCHNEIDER 1996: 39). ${ }^{9}$ Anstatt sie wenigstens anzusprechen, als er sie erreicht, „streckte ich den schon halb erhobenen Arm zu dem verhaßten, nie erlernten Gruß aus, brachte ein englischsportliches ,Hallo!‘ hervor und ging weiter, als hätte ich mich geirrt“" (ebd.). Trotz der Vergewaltigungsphantasie und einem latenten Stalking, liegt in der Szene nichts Relevantes vor, aber das unwillkürliche Heben des Armes interpretiert der Erzähler selbst als Hitlergruß, er sieht sich also hier in einer ungewollten Identität mit dem Vater: Angehöriger einer

\footnotetext{
9 Bei dieser wie bei der folgenden Szene handelt es sich offensichtlich um typische Klischees des südamerikanischen Anderen: hier um die sexualisierte, „exotische“ Frau, dort um die willkürliche und korrupte Staatsmacht.
} 


$$
\text { Galle, H. - Ökonomie der Schuld }
$$

vermeintlich überlegenen Rasse, der einer sozial unterlegenen Person im Affekt Gewalt antun könnte.

In der zweiten Episode kehrt er in das billige Hotel zurück, in dem er nun logiert, und stellt fest, dass die für den Vater bestimmten 500 Dollar aus seiner Brieftasche entwendet wurden. Auf dem Weg kreisten seine Gedanken noch um den Vater („Er ist schuldig! Es muß Gericht gehalten werden!“; SCHNEIDER 1996: 53). Nun verdächtigt er den Hotelier des Diebstahls und empfindet gegen diesen ,einen Haß, den ich nie gekannt hatte“ (SCHNEIDER 1996: 54). Wie sein Vater bei dem „Augenlicht“" seiner Mutter, beschwört der Portier, ein Siebzigjähriger mit einer ,,dunklen Gesichtshaut““(ebd.) bei der Madonna und seinen Kindern seine Unschuld. Die Parallele zur Auseinandersetzung mit dem schuldigen Vater ist überdeutlich. Während der Sohn unfähig ist, den mehr als schuldigen Vater vor Gericht zu bringen, will er nun den vergleichsweise geringfügigen Diebstahl geahndet sehen. Doch der weitere kafkaeske Verlauf zeigt ihm die Aussichtslosigkeit eines solchen Unterfangens in einem von Korruption und Machtprivilegien dominierten Land. Die Polizei macht allem Anschein nach mit dem verdächtigen Hotelier gemeinsame Sache und verhört statt seiner das Zimmermädchen, das offensichtlich schwächste Glied in der sozialen Hierarchie. Die amtlichen Schergen tragen alle Zeichen einer außerhalb der Legalität agierenden, willkürlich Gewalt anwendenden Schlägertruppe, welche die Anzeige als willkommenen Anlass benutzt, um ihr Geschäft an einer Unterschichtfrau auszuüben. Das Schimpfwort, das ihm der beinlose Bettler vor dem Hotel zuzischt, besiegelt die Konversion des Protagonisten in einen Vertreter der Herrenrasse, obwohl er eigentlich nach Brasilien gekommen war, um Rechenschaft von einem Menschheitsverbrecher zu fordern. Auch hier sind die Vorgänge weniger objektiv konstatierbare Sachverhalte als Wahrnehmungen des Protagonisten, die leicht paranoide Züge tragen. Er wird schuldig für relativ banale Dinge, während seine wahre, große Schuld darin bestehen würde, den verbrecherischen Vater nicht gestellt zu haben.

Warum hat Peter Schneider diese Szenen in seine Fiktionalisierung des dokumentierten Besuchs von Rolf Mengele eingefügt? Dieser erweckt in den Interviews nicht den Eindruck, als hätte die Konfrontation mit seinem leiblichen Vater, zu dem er ja nie eine intime, persönliche Beziehung hatte aufbauen können, ihn zu irrationalen Handlungen oder Schuldgefühlen veranlasst hätte. Die Ereignisse lagen immerhin 10 Jahre zurück, als er sie öffentlich machte. Es ist auch nicht so, dass die Öffentlichkeit ihn damals zur 


$$
\text { Galle, H. - Ökonomie der Schuld }
$$

Rechenschaft gezogen hätte, weil er seinen Vater nicht angezeigt und den Gerichten übergeben hat. Juristisch ist das von dem leiblichen Sohn nicht zu verlangen und auch im Rahmen allgemeiner moralischer Erwägungen ist Rolf Mengele wohl nicht vorzuwerfen, er habe sich mit diesem Besuch vor der Menschheit schuldig gemacht. Soweit die Realität.

Aber in der fiktionalen Vergegenwärtigung muss der Protagonist eine tiefe seelische Verzweiflung durchmachen, weil er mit dem Massenmörder konfrontiert ist, und diesen nicht zur Reue oder auch nur einer Schuldanerkenntnis bewegen kann. In dem Dilemma, seinen eigenen Vater nicht umbringen zu können, wie es seinen unmittelbaren Impulsen entspräche, sucht sich das Bestrafungsbedürfnis ein anderes Objekt, den Hotelportier, ohne dass seine Aktionen etwas anderes zur Folge haben, als dass er sich nun selbst schuldig fühlt und in eine analoge Rolle zum Vater geraten ist. Die Logik der Handlung will gewissermaßen sagen: Wer den Menschheitsverbrecher bestrafen könnte und es nicht tut, wird selbst schuldig, auch wenn sich das nicht objektiv begründen lässt.

Wenn wir uns daran erinnern, dass der Autor Peter Schneider von den Rezensenten dafür zur Rechenschaft gezogen wurde, dass er es an Distanz zu seinem Objekt mangeln ließ, so lässt sich hier derselbe irrationale Mechanismus erkennen: Obwohl er für die Taten Josef Mengeles so wenig verantwortlich ist wie dessen Sohn, macht er sich schuldig, weil er nicht zumindest in der Fiktion eine angemessene symbolische „Bestrafung“ des Täters inszeniert hat. Wie der Erzähler aus der Handlung geht der Leser aus der Erzählung mit dem unguten Gefühl des Versagens, der Unzulänglichkeit heraus. Das entspricht dem tatsächlichen Gang der Geschichte, in der Mengele straflos ausgegangen ist und seine Opfer ungesühnt blieben. Er starb in Freiheit, ohne dass sein Sohn oder der Rest der Welt ihn zur Verantwortung gezogen hätten. Das ist ebenso unbefriedigend wie der Verlauf der Erzählung Schneiders. Es bedürfte aber einer kontrafaktischen Fiktion im Stil Tarantinos, um eine solche Schuld gemäß den Erwartungen des Lesers abzugelten.

\section{Literaturverzeichnis}

ADLER, Jeremy D. Die Kunst, Mitleid mit den Mördern zu erzwingen. Einspruch gegen ein Erfolgsbuch: Bernhard Schlinks „Der Vorleser“ betreibt sentimentale Geschichtsfälschung. Süddeutsche Zeitung (20.04.2002). 18. 


$$
\text { Galle, H. - Ökonomie der Schuld }
$$

AMÉRY, Jean. Jenseits von Schuld und Sühne. Bewältigungsversuche eines Überwältigten. In: Werke Band 2. Jenseits von Schuld und Sühne. Unmeisterliche Wanderjahre. Örtlichkeiten. Stuttgart: Klett-Cotta, 2002, p. 7-177.

ARENDT, Hannah. Vita activa oder Vom tätigen Leben. München, Zürich: Piper, 2002.

ARENDT, Hannah. Über das Böse: Eine Vorlesung zu Fragen der Ethik. München, Zürich: Piper, 2015.

ARISTOTELES. Poetik. Griechisch / Deutsch. Stuttgart: Reclam, 1994.

BYHAN, Inge. So entkam mein Vater. Die Geheimnisse des Josef Mengele: Seine Flucht. Seine Verstecke. Seine Jahre im Untergrund. Bunte Illustrierte, Nr. 26, 1986a, 16-33 II.

BYHAN, Inge. So viele halfen ihm. Bunte Illustrierte, Nr. 27, 1986b, p. 17-32.

BYHAN, Inge. Keiner fragte nach seinen Taten. Bunte Illustrierte, Nr. 28, 1986c, p. 26-31.

BYHAN, Inge. Ein Name wie ein Fluch. Bunte Illustrierte, Nr. 29, 1986d, p. 20-31.

BYHAN, Inge. Von Reue keine Spur. Bunte Illustrierte, Nr. 30, 1986e, p. 108-117.

Collins Donahue, William. Illusions of Subtlety: Bernhard Schlink's Der Vorleser and the Moral Limits of Holocaust Fiction. In: German Life and Letters 54, Nr. 1, 2001, p. 60-81.

COLlins DonAHUE, William. Holocaust as fiction. Bernhard Schlink's "Nazi” novels and their films, New York: Palgrave Macmillan, 2010.

Die Hochteutsche RechtsGelahrte SocIETÄT. Allgemeines Juristisches Oraculum: Oder Des Heil. Römisch-Teutschen Reichs Juristen Facultät. Leipzig: Johann Samuel Heinsius, 1747.

GALlE, Helmut. Os escritos autobiográficos de Josef Mengele. Estudos Avançados, Jg. 25, Nr. 71, 2011, p. 269-286.

Hug, Daniel: Katharsis. Revision eines umstrittenen Konzepts, London: Turnshare, 2004.

JANKÉLÉVITCH, Vladimir. Das Verzeihen: Essays zur Moral und Kulturphilosophie. Frankfurt am Main: Suhrkamp, 2004.

JASPERS, Karl. Die Schuldfrage. Von der politischen Haftung Deutschlands (1946). München, Zürich: Piper, 1996.

KRASS, Stephan. Der Rezensionsautomat: Kleine Betriebsanleitung für Leser und Kritiker. Paderborn: Fink, 2012.

LANGBEIN, Hermann. Menschen in Auschwitz. München, Wien: Europaverlag, 1999.

NYISZLI, Miklos. Im Jenseits der Menschlichkeit. Ein Gerichtsmediziner in Auschwitz. Berlin: Dietz, 2005.

Posner, Gerald L.; WARE, John. Mengele. Die Jagd nach dem Todesengel. Berlin: Aufbau, 1993.

RUCH, Philipp. Ehre und Rache. Eine Gefühlsgeschichte des antiken Rechts. Frankfurt, New York: Campus Verlag, 2017.

SCHNEIDER, Peter. Politische Dichtung. Ihre Grenzen und Möglichkeiten. Der Monat. Jg. 17, Nr. 207, 1965, p. 68-77.

SCHNEIDER, Peter: Frühe Manuskriptfassungen zu „Vati“. 1986. AKADEMIE DER KÜNSTE BERLIN, Bestand Peter Schneider, Nr. 1519.

SCHNEIDER, Peter. Vati. Erzählung (1987). München: rororo, 1996.

SCHNEIDER, Peter: Vom Umgang mit dem Bösen in der Literatur. Zur Frage des erzählerischen Abstands. In: LÜTZELER, Paul Michael; KAPCZYNSKI, Jennifer M. (Orgs.). Die Ethik der Literatur: Deutsche Autoren der Gegenwart. Göttingen: Wallstein, 2011, p. 145-156.

Pandaemonium, São Paulo, v. 21, n. 34, maio-ago. 2018, p. 115-133 


\section{Galle, H. - Ökonomie der Schuld}

SCHÖNFELD, Gerda-Marie. Ist Peter Schneiders Erzählung „Vati“ ein schlichtes Illustrierten-Plagiat? Der Spiegel (09.03.1987).

SCHOLZ, Martin. „Bumm, bumm, bumm, bumm“. Quentin Tarantino im Interview. Frankfurter Rundschau (03.08.2009).

SEIDENSTICKER, Bernd. Die Grenzen der Katharsis. In: VÖHLER, Martin; LiNCK, Dirck (Orgs.). Grenzen der Katharsis in den modernen Künsten. Berlin: Walter de Gruyter, 2009, p. 3-20.

SOFSKY, Wolfgang. Die Ordnung des Terrors: Das Konzentrationslager. Frankfurt a. M.: Fischer, 1997.

VöLKLEIN, Ulrich. Josef Mengele. Der Arzt von Auschwitz, Göttingen: Steidl, 1999.

WILDT, Michael. Generation des Unbedingten. Das Führungskorps des Reichssicherheitshauptamtes (2002), Hamburg: Hamburger Edition, 2008.

Recebido em 12 de fevereiro de 2018

Aceito em 20 de fevereiro de 2018 